\title{
Present Day Challenges in Understanding the Geomagnetic Hazard to National Power Grids
}

\author{
A. W. P. Thomson ${ }^{1}$, C. T. Gaunt ${ }^{2}$, P. Cilliers ${ }^{3}$, J. A. Wild ${ }^{4}$, B. Opperman ${ }^{3}$, L.-A.

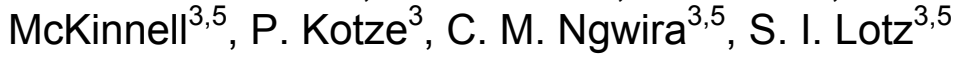 \\ ${ }^{1}$ Geomagnetism, British Geological Survey, West Mains Road, Edinburgh EH9 3LA, UK \\ (awpt@bgs.ac.uk) \\ 2 Department of Electrical Engineering, University of Cape Town, Private Bag, Rondebosch \\ 7701 , South Africa \\ ${ }^{3}$ Space Physics Group, Hermanus Magnetic Observatory, PO Box 32, Hermanus 7200, South \\ Africa \\ ${ }^{4}$ Department of Communication Systems, InfoLab 21, Lancaster University, Lancaster LA1 4WA, \\ UK \\ ${ }^{5}$ Department of Physics and Electronics, Rhodes University, PO Box 94, Grahamstown 6140, South \\ Africa
}

\begin{abstract}
Power grids and pipeline networks at all latitudes are known to be at risk from the natural hazard of geomagnetically induced currents. At a recent workshop in South Africa, UK and South African scientists and engineers discussed the current understanding of this hazard, as it affects major power systems in Europe and Africa. They also summarised, to better inform the public and industry, what can be said with some certainty about the hazard and what research is yet required to develop useful tools for geomagnetic hazard mitigation.
\end{abstract}

\section{Background}

Geomagnetically induced currents (GICs) are a natural hazard that can affect conducting infrastructures, such as power grids and pipelines (e.g. Boteler et al, 1998; Kappenman, 1996, 2004; Pirjola, 2002). GICs are a direct result of solar activity, which gives rise to space weather and consequently to geomagnetic storms. Besides power grids and pipelines, space weather can disrupt communications, use of the global positioning system (GPS), and pose risks to satellite and spacecraft operations. (Recent summaries on the space weather hazard can be found in, for example, Lanzerotti et al, 1999; Pirjola et al, 2005; Thomson, 2007; Eastwood, 2008)

The origin of GICs and space weather lies in the Sun's magnetic activity cycle. The most significant solar phenomenon for space weather is a 'coronal mass ejection' (CME). Heading Earthward in one to three days, CMEs evolve as they interact with the ambient solar wind flow. When CMEs impact the Earth's protective magnetosphere, their energy and electrical plasma boost existing magnetospheric currents. These current systems cause large magnetic variations that induce electric fields in the solid Earth. These fields, in turn, generate the GICs that flow in 
conducting pipes and wires, in ways influenced by the electrical properties of each network.

There is much documented and anecdotal evidence of the effects of GICs on the power systems of the developed world (e.g. Bolduc, 2002; Molinksi, 2002; Pulkkinen et al, 2005; Kappenman, 2005; Trichtchenko et al, 2007). The most widely known example of a damaging impact is the collapse of the Hydro Quebec power system on $13^{\text {th }}$ March 1989. A severe geomagnetic storm shut down the complete high voltage system of Quebec, estimated at within 1 minute and 10 seconds, with a consequent economic cost and social disruption (Bolduc, 2002). More recent storms, for example the October 2003 'Halloween' magnetic storm (which resulted in lower latitude auroral activity, as in Figure 1), are also known to have affected networks in Europe, North America, South Africa and elsewhere (e.g. Pulkkinen et al, 2005; Gaunt and Coetzee, 2007; Thomson et al, 2005). An estimate of the present-day economic cost of a repeat of the most severe geomagnetic storm known (the 'Carrington Storm' of September 1859) has also recently been made by the US National Research Council (2008).

Together with the power grid evidence there is now more than thirty years of scientific research into the subject (including, recently, Erinmez et al, 2002; Kappenman, 2005; Pulkkinen et al, 2005; Wik et al, 2008). Progress has certainly been made, in terms of quantifying the Sun-Earth magnetic connection. However much remains to be done. In those thirty-plus years it has been found that understanding the geomagnetic hazard is a truly cross-disciplinary activity, particularly when considering its impact on technology at ground level. There is therefore much scope remaining for engagement between solar-, space- and geophysicists and the power engineering community, to turn scientific knowledge into practical tools for risk assessment and hazard mitigation.

\section{A Workshop on the GIC Hazard to Power Grids}

In December 2008 the University of Cape Town and the Hermanus Magnetic Observatory hosted a workshop in South Africa for a group of UK and South African scientists. This workshop was funded by the Royal Society, on behalf of the UK government, and by the National Research Foundation, on behalf of the government of South Africa. Using their expertise in space physics, geophysics and electrical power engineering, the workshop participants spent a week discussing issues within the GIC risk to high voltage power grids, in both developed and developing countries around the world. Examples of European and African national power grids were examined.

One aim of the GIC workshop was the free exchange of ideas, insights and knowledge on the natural geomagnetic hazard and on GIC risk. This was for mutual education and to help promote future research between the two countries in the cross-disciplinary manner suggested above. A second aim of the workshop was to summarise the scientific and engineering 'state of play' for the power engineering industry, for the public and for policy makers. The workshop participants therefore compiled a short list of major points that they believed with some confidence that 
scientists and engineers do know about the GIC risk to electric power systems, as well as major things we still don't know.

In the following sections we list, in relatively simple terms, ten major 'do knows' and ten major 'don't knows' about GIC risk, together with some short explanatory notes. The ten items on each of the two lists are ordered approximately in the time-order of CME onset through to power system damage and not in any order of relative importance.

\section{Ten things we do know about the GIC risk to power grids}

1. Solar storms (i.e. CMEs) that lead to high levels of GICs are statistically more likely during periods close to solar maximum and in the descending phase of the solar cycle, but they do also occur at all other times in the solar activity cycle (as identified in Figure 2).

The number of major CMEs varies with the cycle of solar (sunspot) activity. However large amplitude eruptions do occur even when the magnetic activity cycle is at a minimum, for example in 1986. This means that the GIC risk is not restricted to just a few years around the maximum in solar activity. It is always present.

2. The magnetospheric and ionospheric currents that drive GICs are different at different latitudes (see, for example, the complex and dynamic system of ionospheric currents shown in Figure 3 for northern Europe during one major storm).

The Earth's near-space environment (the magnetosphere) contains a complex system of diffuse, but large scale electrical currents that connect with currents that flow in the ionosphere, in the upper atmosphere. These currents are modulated and amplified by solar activity. The structure and dynamics of these current systems changes with latitude. Understanding the details of the behaviour of these current systems, and their effects, is an active research area in space- and geo-physics, though the broad picture is widely agreed. For example, at higher latitudes the 'auroral electrojets' induce largely east-west surface electric fields. In principle, the largest voltage difference across a power network should occur in this orientation. However it turns out to be incorrect to assume that the largest GICs always occur in transmission segments with an east-west orientation; in some cases an almost north-south orientation may be more important.

3. The dominant cause of GICs in power grids is the time rate of change of the Earth's magnetic field.

There are firm theoretical reasons for this, as well as the simple observation of a strong linear correlation between the time-rate-of-change of the magnetic field and measured GICs, at all latitudes. Accurate prediction of GIC risk then requires accurate prediction of changes in the magnetic field 
(see, for example, Wintoft, 2005). This is still scientifically hard to do. (We should note also that recent work has suggested significant correlations between GIC and the field itself: Watari et al, 2009.)

4. Interpolating the magnetic field from spatially distributed geomagnetic observations improves the prediction accuracy of GICs at any given point, even at mid-latitudes (e.g. Bernhardi et al, 2008). This is in comparison with predictions made from data from a single magnetic observatory, taken to be representative of the 'regional' situation.

This follows from point two. The natural magnetic field at any point in a power grid, where we need to predict GICs, is mostly affected by those magnetospheric and ionospheric currents systems that are closest to it. Neighbouring permanent geomagnetic observatories are the best means to interpolate geomagnetic activity to a given measurement site. Simplified assumptions - such as the so-called 'plane-wave model', which is based on single-site data - give an incomplete picture of magnetic changes across regional scale power grids. In addition, measured GIC at any point may be the sum of induction processes in several connected transmission lines.

5. GICs are larger in countries and regions where the geology is generally more resistive (discussed e.g. in Pirjola and Viljanen, 1991).

While the magnitude of the magnetic field change is the most significant variable affecting the magnitude of the GICs, higher resistance rock increases the natural surface electric field that acts as the voltage source (or 'battery') for GICs, operating in the line between the neighbouring grounding points in any given grid (see e.g. Boteler and Pirjola, 1998). Conductivity is a function not just of rock type, but also of fluid and mineral content, and needs to be considered separately for each power network region.

6. A multi-layered and laterally varying ground conductivity model gives better prediction of GICs, than the simpler assumption of an homogeneous Earth (e.g. Ngwira et al, 2008; Thomson et al, 2005).

Closer correlation between measured data and scientific models of GICs is obtained by using a three-dimensional conductivity model of the Earth.

7. GICs have been demonstrated to affect power systems at all latitudes (see, for example, damage to a South African transformer shown in Figure 4).

There is a perception, particularly common within government and industry, that GICs are a risk only for power grids at relatively high latitudes. For example GICs in the past have been widely reported and analysed in Canada, Finland and Scandinavia. GICs have however also been measured and reported in more mid-latitude counties such as the UK and USA. The workshop participants however also learned of GIC effects and studies in South Africa and Brazil, both countries at low geographic and geomagnetic latitudes, and learned of anecdotal evidence for GIC impacts elsewhere in the world, at all latitudes. Recent research papers from Japan (data from 
around 44 degrees north: Watari et al, 2009) and China (around 23 degrees north: Liu et al, 2009) are testament to this. This means that assessing the risk from space weather to our technological infrastructure is a concern for all countries.

8. GICs can affect many power transformers simultaneously at multiple points across regional and continental scale networks (e.g. GIC data in Figure 3).

This distinguishes global and continental scale space weather impacts from localised terrestrial weather impacts such as lightning, ice and severe wind storms. GICs require a different approach to the analysis of power system failure, in comparison with standard approaches that usually assume independence of events initiating system faults ('contingencies').

9. Series capacitors in transmission lines may interrupt GIC flow in power networks, but are expensive. However some strategies involving capacitors may increase GIC and reactive power demands (e.g. Erinmez et al, 2002).

Engineering and mitigation strategies are known to exist that are intended to protect against GIC damage. However this 'do know' tells us that any network as a whole must be protected. Attempting to protect single or just a few assets will merely redistribute GICs and may put other assets at risk. Lower-cost mitigation techniques can be implemented instead of series capacitors in the transmission lines, but these have other effects on power system operation. The capacity to mitigate the impact of GICs might not be within the scope or control of independent and individual owners of transmission networks and power stations. Careful network planning, numerical modelling and testing is suggested in each case.

10. It is possible from transformer dissolved gas analysis to identify GIC-initiated damage before complete transformer failure occurs (see, for example, Figure 5). This is especially true if the rate of gassing simultaneously increases in widely separated transformers across a network.

Excessive heating in a transformer leads to the breakdown of the insulation, releasing gas that can be detected. Tracking the changing level of evolved gas can indicate the risk of future cumulative damage to each transformer, for example while measured GICs remain below warning thresholds.

\section{Ten things we don't know about the GIC risk to power grids}

1. What are the solar and interplanetary events and signatures (peaks, duration, location) that are most 'geo-effective', in terms of GIC causation. For example what significance, if any, can be attached to reported peak solar flare magnitudes, e.g. as listed in Table 1, in understanding historical GIC? 
Scientists have relatively detailed data on solar and interplanetary events going back just 40 years or so. We therefore do not yet know the extremes to which the Sun can operate or know with certainty which particular 'markers' of solar activity are most relevant. Progress on models of solar and solar wind variability is also required.

2. What are the characteristics of extreme geomagnetic storms that pose the highest risk to power systems (see, for example, the relation of major storms to the sunspot cycle shown in Figure 6)?

This is related to point one. Scientists also have relatively detailed and continuous data on geomagnetic events going back to around the 1840s. In many respects the 'Carrington Storm' $\left(1^{\text {st }}\right.$ September 1859) was a far more severe storm than any that has been measured since (see, e.g., US National Research Council, 2008). The possibility of another event of a similar magnitude to this therefore needs to be carefully considered. All available data needs to be fully scanned, digitized and exploited. In particular we note that existing published hourly, daily and annual mean geomagnetic data are not sufficient to fully analyse GIC hazard: the periods of most interest are around a few seconds to a few tens of minutes.

3. In predicting GICs, what is the relative contribution of each of the different components of the geomagnetic field (i.e. the horizontal and vertical components and of the total field magnitude), as well as the relevance of other data, such as the ionospheric total electron content (TEC) and the interplanetary magnetic field magnitude and direction (e.g. Pulkkinen et al, 2006)?

We know that each of these factors is either directly relevant or is a useful proxy for other relevant data, but more study is required. What is the minimum information we need in order to maximise GIC prediction accuracy? Can we, for example, derive useful near real time information on changing ionospheric current systems from GPS TEC measurements that vary with time?

4. What are the definitive spatial and temporal scales of the magnetospheric and ionospheric currents that drive significant GICs in grids?

GICs respond to magnetic variations on many timescales. At this time it is believed that the dominant periods of interest are probably a few seconds to tens of minutes. How detailed do our ionospheric and magnetospheric current models therefore need to be, in time and space, to capture the physics of the problem?

5. What is an adequate number and distribution of magnetometers for GIC modeling in the UK and South Africa (and similarly for other countries)?

This is related to point four. Can we define guidelines that help developed and developing countries, wherever they are, to set up networks of magnetometers; or do we need to approach the problem on a case by case basis, with regard to latitude, geology, geophysics and space physics? 
6. Which information, given on what timescale, is most useful for any given power utility/ authority to manage its $\mathrm{GIC}$ risk?

CMEs take between just under one day and perhaps up to 3-4 days to reach the Earth. The solar physics underpinning CME formation and release is not well understood and predictive models of CMEs are limited in their capabilities. So much is still required scientifically. However the question can still be posed of industry: what would be ideal and what would be desirable in terms of warning time and information content? Also, what are the costs and other consequences of incorrect high-activity forecasts?

7. In modeling GICs in a power grid, what is an appropriate level of detail required of Earth conductivity (as a three dimensional model or otherwise)?

In practical terms simple conductivity models that vary only with depth remain useful for inland continental regions, away from coasts. But what about the coasts, where many high-value generating assets are located, due to proximity to cooling water and access to fuel? How accurate does industry need GIC modeling to be? How quickly do models have to perform to provide useful forecast data, and does this imply restricting complexity of model to meet targets?

8. What are the characteristics of power transformers that determine their susceptibility to GICs and therefore determine the extent of damage sustained under different levels of GICs?

Industry data on transformer designs need to be examined in more detail (e.g. Lahtinen and Elovaara, 2002). How can transformers be tested or certified for compliance with specifications that are intended to reduce damage by GICs?

9. What are the transformer failure mechanisms subsequent to damage initiated by GICs?

This is related to point eight. Major details are understood from observations of $\mathrm{GIC}$ damage, but laboratory tests on failure modes are rare. How do gassing and other condition monitoring records help? Are there emerging industry standards for transformers, for protection against GICs and the subsequent deterioration leading to failure? What other work needs to be done here?

10. Where should scientists go to gain access to industry archives, particularly archives of any $\mathrm{GIC}$ measurements obtained concurrently with network data (i.e. network configuration and connections, DC resistances of transmission lines and transformers and station earthing resistances)?

The absence of open source data is a continuing problem for scientists, although the commercial issues are appreciated. However some progress, perhaps through support from national industry regulators, industry or 
professional societies would be helpful. Also, there is a need for long term monitoring in any given power grid, as the electrical (near-DC)

characteristics of each grid changes over time.

\section{Conclusions}

Compared with the 'do knows' in our list, our 'don't knows' may be more contentious within the scientific community. It may be debated which items are most important at the present time, understanding that other issues might yet become more relevant. However by making scientific progress on our current 'don't knows' we do believe that scientists will improve their ability to monitor, model and predict the impacts of space weather and GICs on power grids.

Solar cycle 24 is just beginning and we can expect that the space weather hazard to ground-based technologies will increase, just as it did during the up-turn of previous cycles. The main message of the Hermanus workshop is therefore that we need a wider discussion on all of the issues, not just within the GIC science community, but also within industry and wider society. We hope that our lists will go some way to start that discussion and promote much needed future research.

A future scientific workshop is planned for 2010/2011, again in South Africa. This meeting is planned to be more broadly based than the recent one was, involving invitations to specialists from outside the UK and South Africa. At this meeting we will discuss what progress has been made against our list of 'don't knows' and, in general, discuss progress on GIC risk assessment and on the geomagnetic hazard. We would also like to be able to consider the wider impacts of GIC, for example within pipeline and railway networks.

\section{Acknowledgements}

The workshop was supported financially by a 'UK-South Africa Science Network Phase II' grant. Travel costs were met by the Royal Society and local costs in South Africa by the National Research Foundation. The attendance of all the participants was supported by their respective institutions. Magnetic and GIC data supplied by geomagnetic observatories, variometer networks and power companies is gratefully acknowledged. Ellen Clarke (British Geological Survey) is also thanked for helping prepare Figure 6. This paper is published with permission of the Director, British Geological Survey (NERC). 


\section{References}

Bernhardi, E.H., Cilliers, P.J., and Gaunt, C.T. (2008). Improvement in the modelling of geomagnetically induced currents in South Africa. South African Journal of Science. 104, July/August, 265-272.

Bolduc, L. (2002). GIC observations and studies in the Hydro-Quebec power system. J. Atmos. Sol. Terr. Phys. 64, 1793-1802. (doi:10.1016/S13646826(02)00128-1)

Boteler, D.H., Pirjola, R.J., and Nevanlinna, H. (1998). The effects of geomagnetic disturbances on electrical systems at the Earth's surface. Advances in Space Research, 22(1): 17-27.

Boteler, D. H. and R. J. Pirjola (1998). Modelling Geomagnetically Induced Currents produced by Realistic and Uniform Electric Fields. IEEE Transactions on Power Delivery, Vol. 13, No. 4, pp. 1303-1308.

Eastwood, J.P. (2008). The science of space weather, Phil. Trans. R. Soc. A 366, 4489-4500. (doi:10.1098/rsta.2008.0161)

Erinmez, I. A., J. G. Kappenman, W. A. Radasky (2002). Management of the geomagnetically induced current risks on the National Grid Company's electric power transmission system. J. Atmos. Sol. Terr. Phys., 64, p743756.

Gaunt, C.T. and Coetzee, G. (2007). Transformer failures in regions incorrectly considered to have low GIC-risk. In Proc. IEEE Powertech Conference, July 2007, Lausanne, Switzerland.

Kappenman, J. G. (1996). Geomagnetic storms and their impact on power systems, IEEE Power Eng. Rev., May 1996, 5-8.

Kappenman, J. G. (2004). An Overview of the Increasing Vulnerability Trends of Modern Electric Power Grid Infrastructures and the potential consequences of Extreme Space Weather Environments, in Effects of Space Weather on Technology Infrastructure, edited by I. A. Daglis, 257-286, NATO Science Series. II. Mathematics, Physics and Chemistry, 176, Chapter 14: Space Weather and the Vulnerability of Electric Power Grids. Kluwer Academic Publishers, Dordrecht, Netherlands.

Kappenman, J. G. (2005). An overview of the impulsive geomagnetic field disturbances and power grid impacts associated with the violent Sun-Earth connection events of 29-31 October 2003 and a comparative evaluation with other contemporary storms. Space Weather 3, S08 C01.

(doi:10.1029/2004SW000128). 
Lahtinen, M. and J. Elovaara (2002). GIC Occurrences and GIC Test for $400 \mathrm{kV}$ System Transformer, IEEE Transactions on Power Delivery, 17, No. 2, pp. 555-561.

Lanzerotti, L. J., Thomson, D. J., and Maclennan, C. G. (1999). Engineering issues in space weather, in Modern Radio Science 1999, edited by M. A. Stuchly, 25-50, International Union of Radio Science (URSI), Oxford University Press, Oxford, UK.

Liu, C.-M., L.-G. Liu, R. Pirjola, and Z.-Z. Wang (2009), Calculation of geomagnetically induced currents in mid to low-latitude power grids based on the plane wave method: A preliminary case study, Space Weather, 7, S04005, doi:10.1029/2008SW000439.

Molinski, T. S. (2002). Why utilities respect geomagnetically induced currents, $J$. Atmos. Sol.-Terr. Phys., 64(16), 1765-1778.

Ngwira, C. M., A. A. Pulkkinen, L.-A. McKinnell, and P. J. Cilliers, Improved modeling of geomagnetically induced currents in the South African power network, Space Weather, 6, S11004, doi:10.1029/2008SW000408, 2008.

Pirjola, R. (2002). Geomagnetic effects on ground-based technological systems. Surv. Geophys. 23, 71-90.

Pirjola, R., Kauristie, K., Lappalainen, H., Viljanen, A. and Pulkkinen, A. (2005). Space weather risk. Space Weather, 3, S02A02.

Pirjola, R. J. and A. T. Viljanen (1991). Geomagnetic Induction in the Finnish 400 kV Power System. Environmental and Space Electromagnetics, Proceedings of the International URSI Symposium on Environmental and Space Electromagnetics, Tokyo, Japan, September 4-6, 1989, edited by H. Kikuchi, Springer-Verlag, Tokyo, Printed in Hong Kong, Chapter 6.4, pp. 276-287.

Pulkkinen, A., Lindahl, S., Viljanen, A. , and Pirjola, R. (2005). Geomagnetic storm of 29-31 October 2003: geomagnetically induced currents and their relation to problems in the Swedish high voltage power transmission system. Space Weather 3, S08C03. (doi:10.1029/2004SW000123)

Pulkkinen, A., A. Viljanen and R. Pirjola (2006). Estimation of geomagnetically induced current levels from different input data. Space Weather, 4, No. 8, S08005, doi: 10.1029/2006SW000229

Thomson, A. W. P., McKay, A. J., Clarke, E. and Reay, S. J. (2005). Surface electric fields and geomagnetically induced currents in the Scottish Power grid during the 30 October 2003 geomagnetic storm. Space Weather 3, S11002. (doi:10.1029/2005SW000156)

Thomson, A.W.P. (2007), Geomagnetic Hazards, in Encyclopaedia of Geomagnetism and Paleomagnetism, p316-319, eds Gubbins, D. and 
Herrero-Bervera, E., Springer, Dordrect, Netherlands. ISBN-13: 978-1-40203992-8.

Trichtchenko, L., A. Zhukov, R. van der Linden, S. M. Stankov, N. Jakowski, I. Stanisławska, G. Juchnikowski, P. Wilkinson, G. Patterson, and A. W. P. Thomson (2007), November 2004 space weather events: Real-time observations and forecasts, Space Weather, 5, S06001, doi:10.1029/2006SW000281.

US National Research Council (2008), Severe Space Weather Events Understanding Societal and Economic Impacts. Committee on the Societal and Economic Impacts of Severe Space Weather Events: A Workshop. Space Studies Board, National Research Council. National Academies Press, Washington, DC 20055, USA, ISBN-13: 978-0-309-12769-1.

Watari, S., M. Kunitake, K. Kitamura, T. Hori, T. Kikuchi, K. Shiokawa, N. Nishitani, R. Kataoka, Y. Kamide, T. Aso, Y. Watanabe,Y. Tsuneta (2009), Measurements of geomagnetically induced current in a power grid in Hokkaido, Japan, Space Weather, 7, S03002, doi:10.1029/2008SW000417

Wik, M., A. Viljanen, R. Pirjola, A. Pulkkinen, P. Wintoft, and H. Lundstedt, Calculation of geomagnetically induced currents in the $400 \mathrm{kV}$ power grid in southern Sweden, Space Weather, 6, S07005, doi:10.1029/2007SW000343, 11 pp., 2008.

Wintoft, P., Study of the solar wind coupling to the time difference horizontal geomagnetic field, Annales Geophysicae, 23, pp. 1949 - 1957, 2005. 


\section{Tables}

\begin{tabular}{|c|c|c|}
\hline Rank & Year/Month/Day & X-Ray Class \\
\hline 1 & $2003 / 11 / 04$ & $\mathrm{X} 28+$ \\
\hline 2 & $2001 / 04 / 02$ & X20.0 \\
\hline 2 & $1989 / 08 / 16$ & $\mathrm{X} 20.0$ \\
\hline 3 & $2003 / 10 / 28$ & $\mathrm{X} 17.2$ \\
\hline 4 & $2005 / 09 / 07$ & $\mathrm{X} 17$ \\
\hline 5 & $1989 / 03 / 06$ & X15.0 \\
\hline 5 & 1978/07/11 & $\mathrm{X} 15.0$ \\
\hline 6 & $2001 / 04 / 15$ & $\mathrm{X} 14.4$ \\
\hline 7 & $1984 / 04 / 24$ & $\mathrm{X} 13.0$ \\
\hline 7 & $1989 / 10 / 19$ & X13.0 \\
\hline 8 & $1982 / 12 / 15$ & $\mathrm{X} 12.9$ \\
\hline 9 & $1982 / 06 / 06$ & $\mathrm{X} 12.0$ \\
\hline 9 & 1991/06/01 & X12.0 \\
\hline 9 & 1991/06/04 & $\mathrm{X} 12.0$ \\
\hline 9 & $1991 / 06 / 06$ & $\mathrm{X} 12.0$ \\
\hline 9 & $1991 / 06 / 11$ & $\mathrm{X} 12.0$ \\
\hline 9 & $1991 / 06 / 15$ & X12.0 \\
\hline 10 & $1982 / 12 / 17$ & $\mathrm{X} 10.1$ \\
\hline 10 & $1984 / 05 / 20$ & $\mathrm{X} 10.1$ \\
\hline 11 & $2003 / 10 / 29$ & $\mathrm{X} 10$ \\
\hline 11 & $1991 / 01 / 25$ & $\mathrm{X} 10.0$ \\
\hline 11 & 1991/06/09 & $\mathrm{X} 10.0$ \\
\hline 12 & $1982 / 07 / 09$ & X9.8 \\
\hline 12 & $1989 / 09 / 29$ & $\mathrm{X} 9.8$ \\
\hline 13 & $1991 / 03 / 22$ & $\times 9.4$ \\
\hline 13 & $1997 / 11 / 06$ & $\mathrm{X} 9.4$ \\
\hline 14 & $1990 / 05 / 24$ & $\times 9.3$ \\
\hline 15 & $2006 / 12 / 05$ & X9 \\
\hline 15 & 1980/11/06 & $\times 9$ \\
\hline 15 & $1992 / 11 / 02$ & $\mathrm{X} 9$ \\
\hline
\end{tabular}

Table 1 The most powerful solar flares recorded since 1976. Those shown in boldface are post year 2000. 'X-Ray Class' denotes flare magnitude in units of $10^{-4}$ $\mathrm{W} / \mathrm{m}^{2}$, between one and eight Angstroms wavelength.

Source: http://spaceweather.com/solarflares/topflares.html 


\section{Figures}
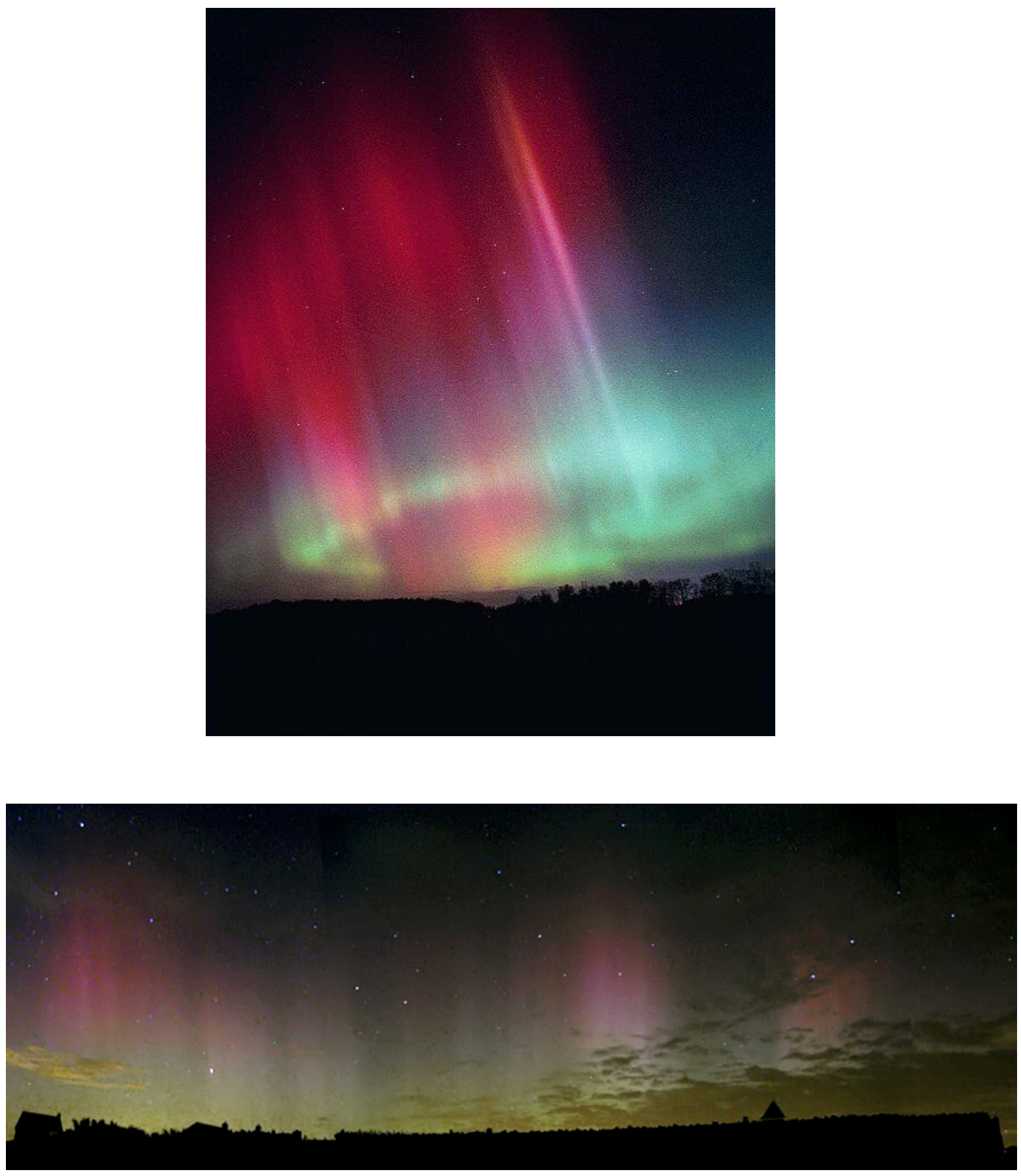

Figure 1 Top: Aurora near LaOtto, Indiana, USA (41.29 geographic north) photographed on 29 October at the time when the CME that emanated from sunspot 486 at 12:18 UT on 28 October 2003 hit Earth's magnetic field, triggered an extreme geomagnetic storm and pushed the auroral oval as far south as Texas. (Photo credit: Robert B. Slobins, as submitted to www.Spaceweather.com). Bottom: A related later auroral event seen from Selsey, in the south of England (at $47.2^{\circ} \mathrm{N}$ magnetic north) taken on 31 Oct 2003. (Photo credit: Pete Lawrence, www.digitalsky.org.uk). 


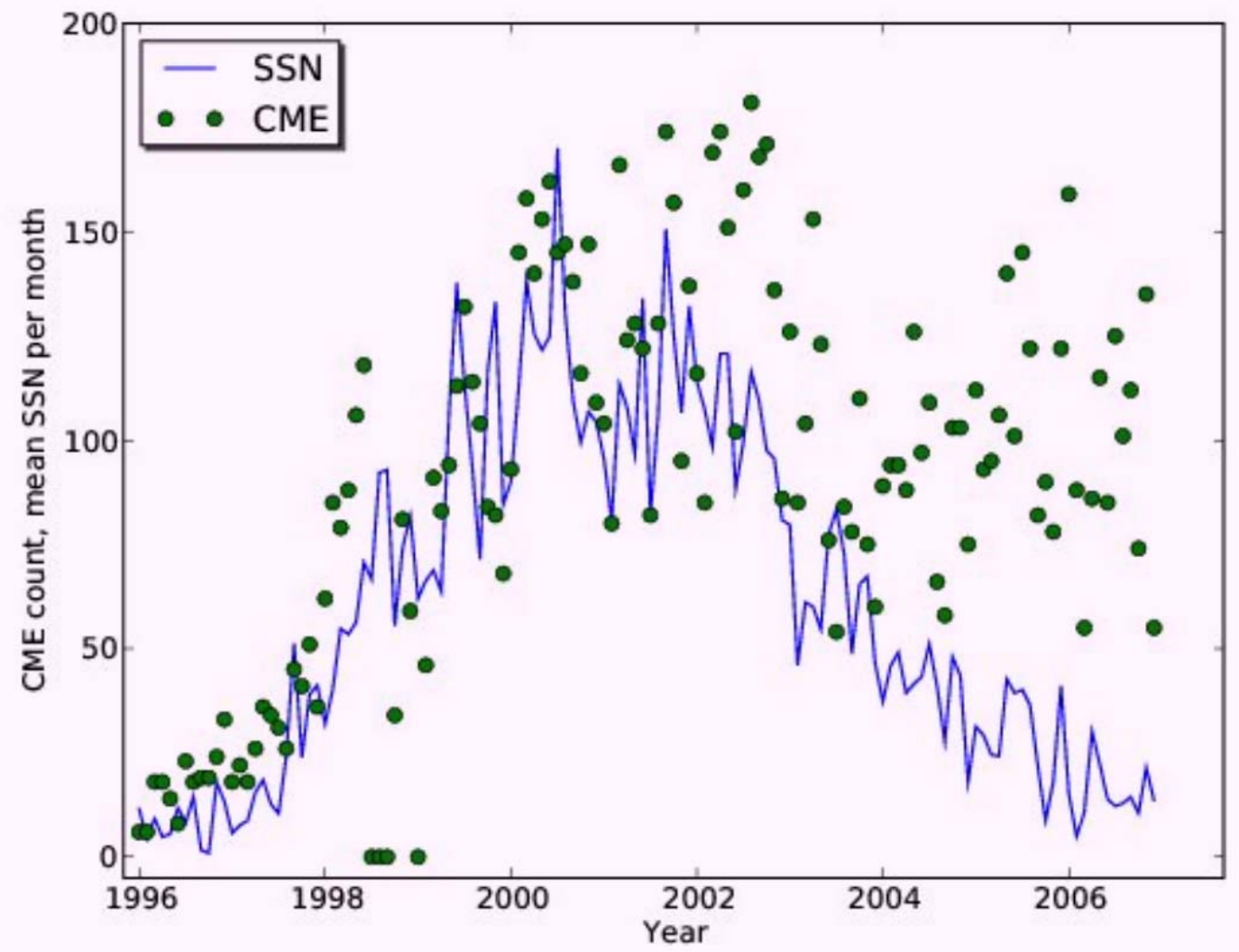

Figure 2 The monthly sunspot number (blue line) and monthly CME count during solar cycle 23. The number of CMEs (and consequent geomagnetic activity) tends to follow the sunspot cycle but remains high even as the sunspot number declines. There are only rarely times when the CME count drops to zero or, equivalently, when the likelihood of major magnetic storms disappears. (Sunspot data courtesy of NGDC/NOAA. CME data courtesy of NASA/ESA SOHO/LASCO). 


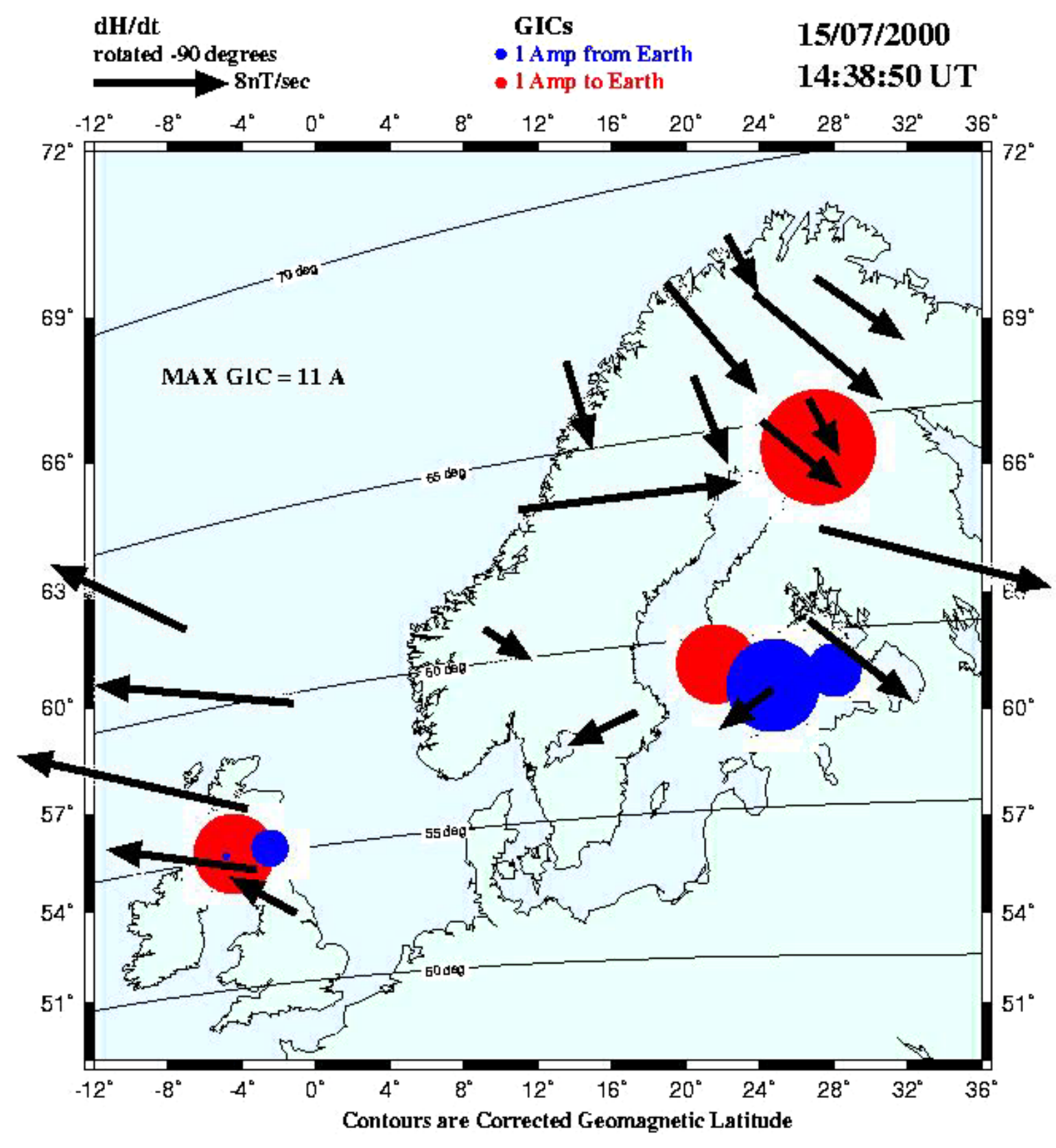

Figure 3 The complexity of ionospheric current vectors are shown, derived from UK and Scandinavian magnetometer data near the time of the storm commencement during the July $15^{\text {th }} 2000$ magnetic storm. The scale vector at top left is $8 \mathrm{nT} / \mathrm{sec}$. Coloured spots denote measured GIC at six points in national power grids at the time (red denotes a GIC flowing to the Earth) and in one gas pipeline in Finland. Spot size is proportional to measured current: the largest current in this image was around $11 \mathrm{~A}$ at the time. Data are courtesy of Finnish Meteorological Institute (IMAGE), Lancaster University (SAMNET), British Geological Survey, Scottish Power plc and Gasum Oy. 


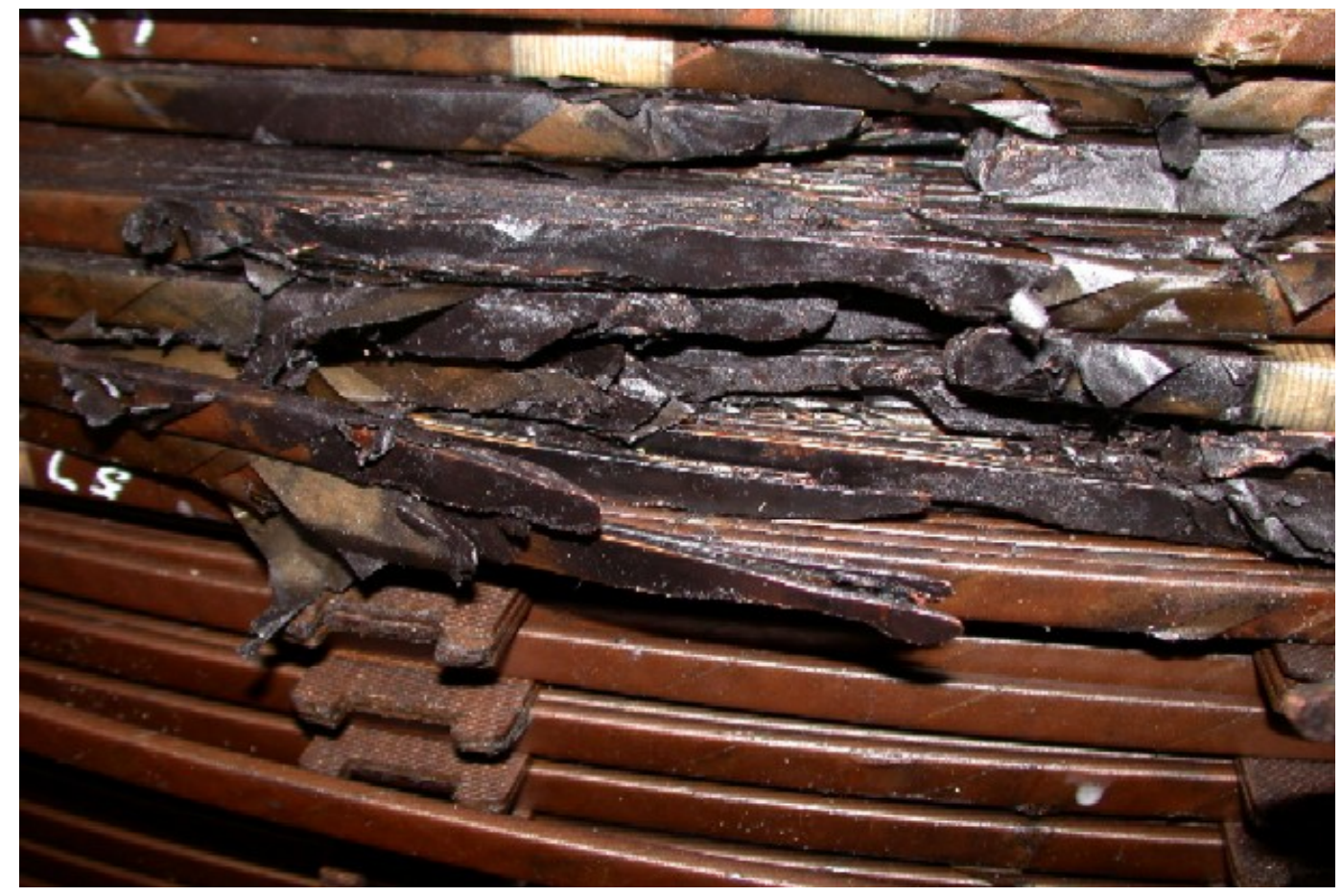

Figure 4 Failure in a large South African generator transformer three weeks after the Halloween storm of October 2003. The disruption of the winding and insulation by the arcing fault at the time of final failure is clear. The arcing fault also destroys evidence that might lead to a better understanding of the progression of damage after initiation by the geomagnetic current event. 


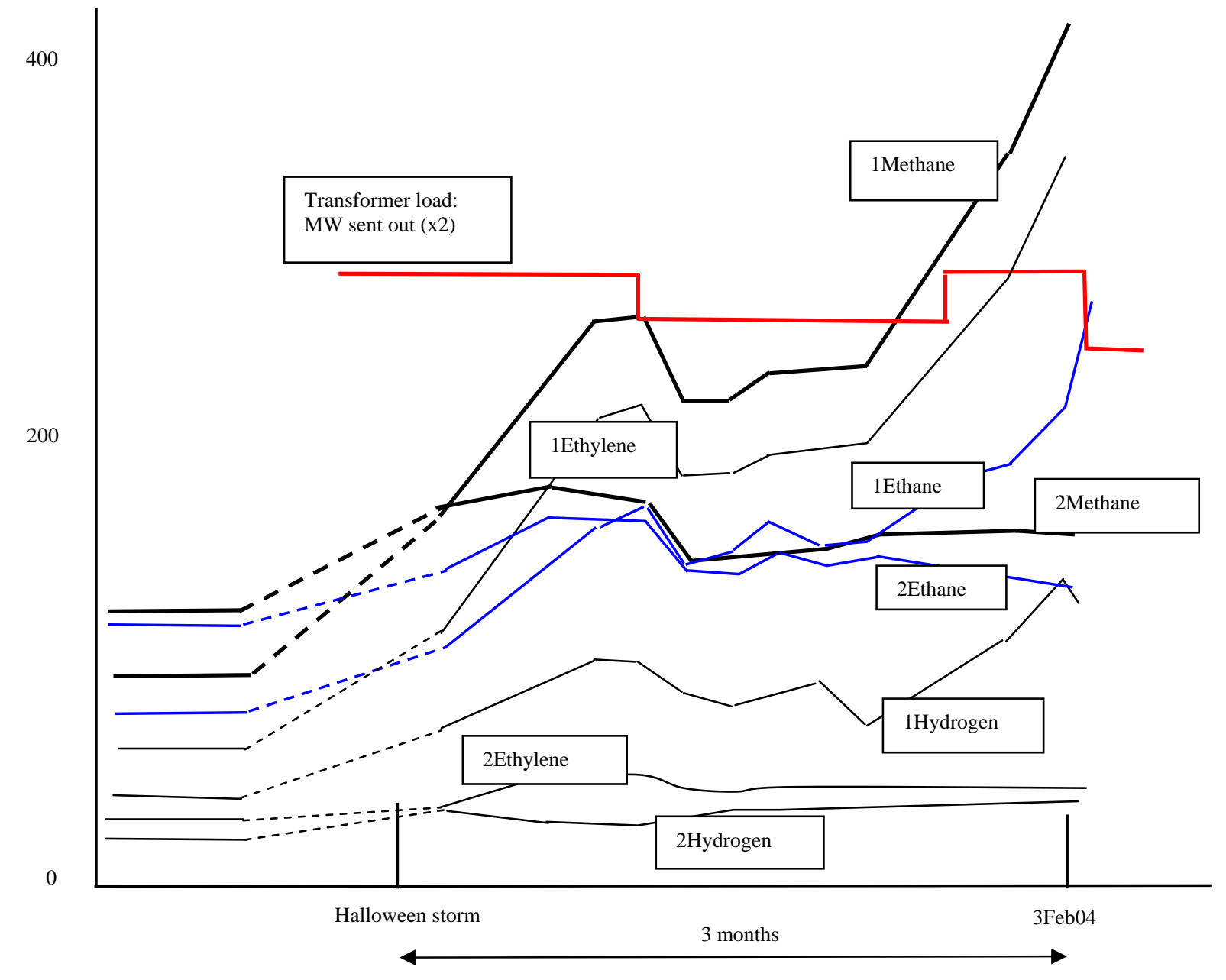

Figure 5 Results of dissolved gas analysis (ppm - left scale) for two similar generator transformers in South Africa (labelled 1 and 2). This illustrates continued gas generation after the short geomagnetic storm (only a few hours) and an apparent sensitivity to transformer loading (MW - same scale) during the following months. The ratios of the different gases also indicate low temperature degradation of paper insulation. Both transformers were removed from service approximately six months after the storm. Note: the dashed lines indicate the increase from nominal pre- to immediate post-storm levels for each data type. 
Sunspot Cycle and Maximum aa* in each Magnetic Storm

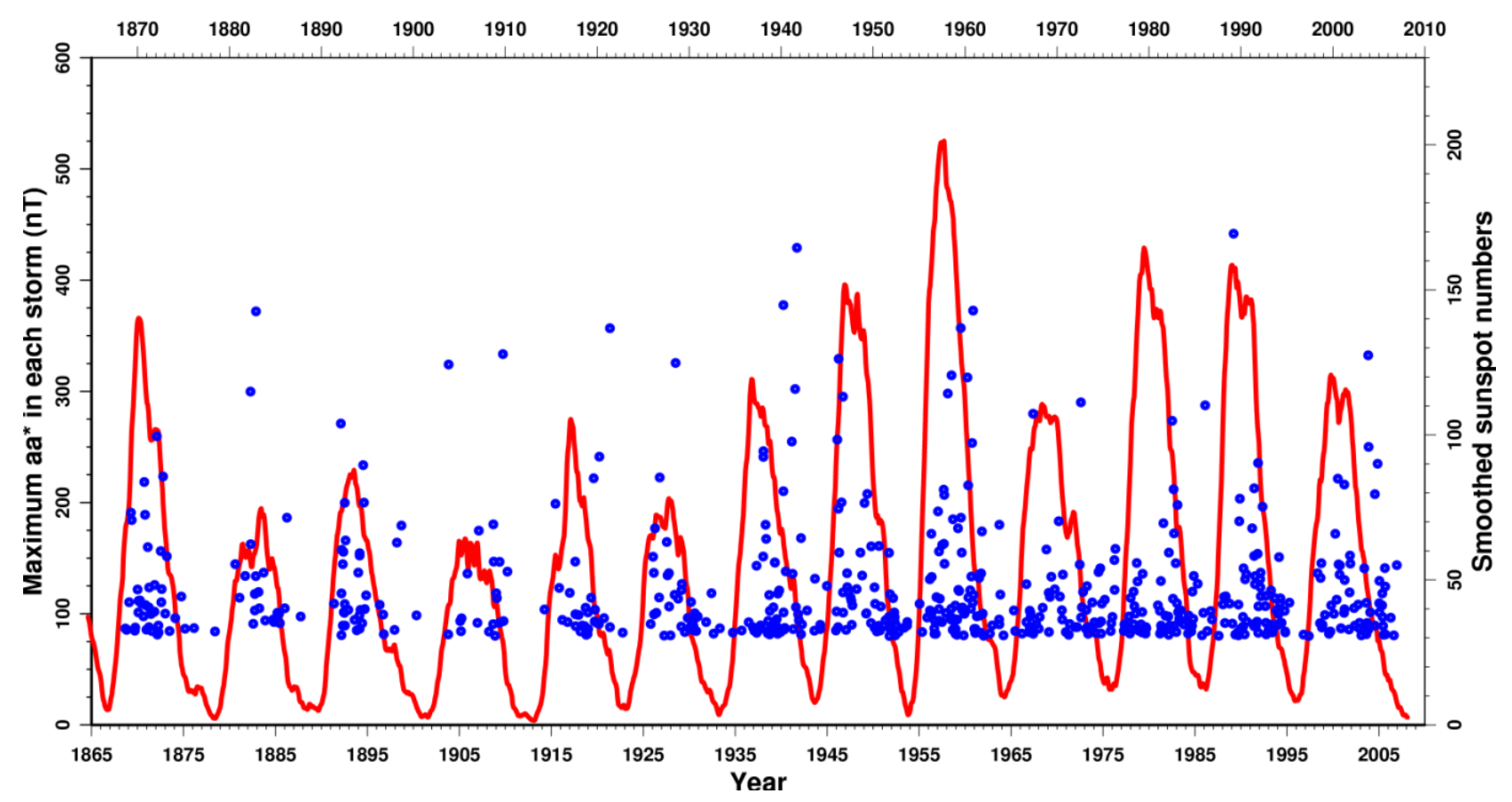

Figure 6 Large storms identified by the peak in the 24-hour running average $\left(a a^{*}\right)$ of the 3-hour geomagnetic aa index against time, since 1868, overlain with monthly smoothed solar sunspot number. A threshold of $80 \mathrm{nT}$ has been used to better identify the largest individual storms. 